\title{
Hospitalization potential at an infectious diseases division of a metropolitan Hospital of Northern Italy; persisting limitations and related problems
}

Roberto Manfredi

From $16^{\text {th }}$ International Symposium on HIV and Emerging Infectious Diseases

Marseille, France. 24-26 March 2010

\section{Background}

Notwithstanding the deep modifications of the natural history of HIV/AIDS, the hospitalization potential of Infectious Diseases (ID) wards remains largely inadequate in Italy, according to the continued modification of epidemiology and disease spectrum.

\section{Methods}

A surveillance study of patients (p) needing hospitalization at our inpatient ward (located at S. Orsola Hospital, Bologna, Italy) and their outcome, was prospectively performed.

\section{Results}

From January 2000 to May 2002 our inpatient unit could rely on 16 beds, while since June 2003 (after joining with the other ID unit of our 800,000-inhabitant metropolitan area), the available beds rose to 35 . The ID Specialist must act as a consultant for every p with a suspected ID, to assess need of hospitalization and/or isolation measures, and eventually search an adequate place (the so-called "bed service"), should room is not available at our ward. The rate of $\mathrm{p}$ admitted elsewhere dropped from the year 2000 (34.3\%), to 2001 (26.9\%), and $2002(12.9 \%)$, but reached a stabilization during years $2003-2008$ (12.1\%; $p<.0001$ versus year 2000). Among the $771 \mathrm{p}$ who could not be admitted by us, no epidemiologic differences were found during time, and HIV disease prevailed (311 p:40.3\%). When infectious (but not diffusive) illnesses are of concern, $\mathrm{p}$ may be accepted by other city Hospitals, while the event of diffusive ID required a transfert to the closest ID ward.
Until May 2002, the other city ID Unit accepted over $30 \%$ of $\mathrm{p}$, but the unification into a single ward partially reduced the need of transferts $40-115 \mathrm{Km}$ far from our city, stabilizing the rate around $12-13 \%$ in the last six years. Among the 311 HIV p not accepted at our ward, $26 \%$ had a place at the other ID ward, $61 \%$ at our Hospital, $3 \%$ at Hospitals of the Bologna province, while $10 \%$ needed a transfert to other cities.

\section{Discussion}

Still in the third millennium, ID wards play a key role in health care inpatient assistance, although a continuous fitting to prevailing ID and available resources is needed. The lack of suitable beds for $\mathrm{p}$ needing admission remains a striking problem, especially when $\mathrm{p}$ with acute-severe illness are of concern, and a long-distance transfert may led to potentially severe risks for $\mathrm{p}$ health, and breakdown of isolation/protection measures.

Published: 11 May 2010

doi:10.1186/1742-4690-7-S1-P159

Cite this article as: Manfredi: Hospitalization potential at an infectious diseases division of a metropolitan Hospital of Northern Italy; persisting limitations and related problems. Retrovirology 2010 7(Suppl 1):P159.

Correspondence: Roberto.manfredi@unibo.it

Infectious Diseases, University of Bologna, S. Orsola Hospital, Bologna, Italy 